PROCEEDINGS OF THE

AMERICAN MATHEMATICAL SOCIETY

Volume 135, Number 1, January 2007, Pages 135-139

S 0002-9939(06)08770-3

Article electronically published on August 16, 2006

\title{
FENCHEL DUALITY, FITZPATRICK FUNCTIONS AND THE EXTENSION OF FIRMLY NONEXPANSIVE MAPPINGS
}

\author{
HEINZ H. BAUSCHKE
}

(Communicated by Jonathan M. Borwein)

\begin{abstract}
Recently, S. Reich and S. Simons provided a novel proof of the Kirszbraun-Valentine extension theorem using Fenchel duality and Fitzpatrick functions. In the same spirit, we provide a new proof of an extension result for firmly nonexpansive mappings with an optimally localized range.
\end{abstract}

Throughout this paper, we assume that $X$ is a real Hilbert space, with inner product $p=\langle\cdot \mid \cdot\rangle$ and induced norm $\|\cdot\|$, and we denote the identity mapping on $X$ by Id. A mapping $T$ from a subset $D$ of $X$ to $X$ is called firmly nonexpansive if

$$
(\forall x \in D)(\forall y \in D) \quad\|T x-T y\|^{2}+\|(\operatorname{Id}-T) x-(\operatorname{Id}-T) y\|^{2} \leq\|x-y\|^{2} ;
$$

equivalently [13, 14, if $2 T$ - Id is nonexpansive (Lipschitz continuous with constant 1), i.e.,

$$
(\forall x \in D)(\forall y \in D) \quad\|(2 T-\mathrm{Id}) x-(2 T-\mathrm{Id}) y\| \leq\|x-y\|
$$

or if

$$
(\forall x \in D)(\forall y \in D) \quad 0 \leq\langle T x-T y \mid(\operatorname{Id}-T) x-(\operatorname{Id}-T) y\rangle
$$

Firmly nonexpansive mappings play an important role in various contexts; see, e.g., 1, 2, 3, 7, 8, 9, 10, 15, 17, 21, 22, 25. The Kirszbraun-Valentine theorem (see, e.g., [5, 13, 16, 20, 26]) states that any nonexpansive mapping can be extended to a nonexpansive mapping defined on the whole space. A beautiful proof of this result, based on Fenchel duality and Fitzpatrick functions, was recently provided by Reich and Simons [23. (For further applications of Fitzpatrick functions, see, e.g., 4, 24.) In this note, we refine their technique to obtain a new proof of an extension theorem for firmly nonexpansive mappings where the range of the extension is optimally localized. This extension theorem easily implies the Kirszbraun-Valentine result. Notation not explicitly defined in the following is standard in convex analysis; see, e.g., [27].

Received by the editors July 24, 2005.

2000 Mathematics Subject Classification. Primary 46C05, 47H09; Secondary 52A41, 90C25.

Key words and phrases. Fenchel duality, firmly nonexpansive mapping, Fitzpatrick function, Kirszbraun-Valentine theorem.

(C)2006 American Mathematical Society Reverts to public domain 28 years from publication 
Definition 1. Let $D$ be a nonempty subset of $X$ and let $T: D \rightarrow X$ be firmly nonexpansive. Then the associated Fitzpatrick function [12] $\phi=\phi_{T}$ is

$$
X \times X \rightarrow]-\infty,+\infty]:(x, y) \mapsto \sup _{d \in D}\langle x \mid d-T d\rangle+\langle y \mid T d\rangle-\langle T d \mid d-T d\rangle,
$$

and we also set $G=G_{T}=\{(d-T d, T d) \mid d \in D\}$.

Proposition 2. Let $D$ be a nonempty subset of $X$, let $T: D \rightarrow X$ be firmly nonexpansive, and let $x$ and $y$ be in $X$. Then:

(i) $\phi=\left(\iota_{G}+p\right)^{*}$.

(ii) The extension $\widetilde{T}: D \cup\{y\} \rightarrow X$ of $T$ which maps $y$ to $x$ is still firmly nonexpansive if and only if $\phi(y-x, x) \leq p(y-x, x)$.

(iii) $\phi$ is convex, lower semicontinuous and proper.

(iv) conv $G \subset \operatorname{dom} \phi^{*} \subset \overline{\mathrm{conv}} G \subset \overline{\mathrm{conv}}(\mathrm{Id}-T)(D) \times \overline{\mathrm{conv}} T(D)$.

(v) $p \leq \phi^{*}$.

Proof. Fix $x$ and $y$ in $X$. (i) For every $d \in D$, we have

$\langle x \mid d-T d\rangle+\langle y \mid T d\rangle-\langle d-T d \mid T d\rangle=\langle(d-T d, T d) \mid(x, y)\rangle-\left(p+\iota_{G}\right)(d-T d, T d)$,

from which the identity follows by supremizing over $d \in D$. (ii) This is a consequence of (3). (iii). By (ii), $\phi \leq p$ on $G$. Hence $\phi$ is proper. The function $\phi$ is convex and lower semicontinuous, as it is a Fenchel conjugate by (i). (iv). This is clear, since $G=\operatorname{dom}\left(\iota_{G}+p\right)$ and $\phi=\left(\iota_{G}+p\right)^{*}$. (v). In view of (3), $p(G-G) \subset[0,+\infty[$. Suppose that $(x, y) \in \operatorname{conv} G$, say it is a finite convex combination $(x, y)=\sum_{i \in I} \lambda_{i}\left(x_{i}, y_{i}\right)$ of elements in $G$. Then $\sum_{i \in I} \lambda_{i} p\left(x_{i}, y_{i}\right)=$ $p(x, y)+\frac{1}{2} \sum_{i, j \in I} p\left(\left(x_{i}, y_{i}\right)-\left(x_{j}, y_{j}\right)\right) \geq p(x, y)$, hence $p \leq \operatorname{conv}\left(\iota_{G}+p\right)$. Since $p$ is continuous, it follows that $p \leq \overline{\operatorname{conv}}\left(\iota_{G}+p\right)=\left(\iota_{G}+p\right)^{* *}=\phi^{*}$.

Fact 3 (Fenchel duality). Let $Y$ be a real Hilbert space and let $L: Y \rightarrow X$ be linear and continuous. Let $f: Y \rightarrow]-\infty,+\infty]$ and $g: X \rightarrow]-\infty,+\infty]$ be convex, lower semicontinuous, and proper such that $g$ is continuous and finite at some point in $L \operatorname{dom} f$. Then

$$
\inf _{y \in Y}(f(y)+g(L y))=-\min _{x \in X}\left(f^{*}\left(-L^{*} x\right)+g^{*}(x)\right) .
$$

Proof. See, e.g., [27, Corollary 2.8.5].

Theorem 4. Let $D$ be a nonempty subset of $X$, let $T: D \rightarrow X$ be firmly nonexpansive, and let $y \in X$. Then $T$ has a firmly nonexpansive extension $\widetilde{T}: D \cup\{y\} \rightarrow$ $\overline{\mathrm{conv}} T(D)$.

Proof. Set $\phi=\phi_{T}$ and $C=\overline{\operatorname{conv}} T(D)$, and assume first that $y=0$. In view of Proposition 2[(ii)] we must show that

$$
\min _{x \in X} \phi(x,-x)+\|x\|^{2}+\iota_{C}(x) \leq 0 .
$$

Set $f: X \times X \rightarrow]-\infty,+\infty]:\left(x^{*}, y^{*}\right) \mapsto \frac{1}{2} \phi^{*}\left(2 x^{*}, 2 y^{*}\right)$ so that $f^{*}=\frac{1}{2} \phi$, and let $j=\frac{1}{2}\|\cdot\|^{2}$. Now set $g=\left(j+\iota_{C}\right)^{*}$ and observe (using [19]) that $g=j \square \iota_{C}^{*}=$ $j-\left(j \square \iota_{C}\right)=j-\frac{1}{2} d_{C}^{2}$, where $\square$ denotes the infimal convolution and $d_{C}$ the distance function. Further set $L: X \times X \rightarrow X:\left(x^{*}, y^{*}\right) \mapsto y^{*}-x^{*}$. We claim that $\inf _{\left(x^{*}, y^{*}\right) \in X \times X} f\left(x^{*}, y^{*}\right)+g\left(L\left(x^{*}, y^{*}\right)\right) \geq 0$. Indeed, pick $\left(x^{*}, y^{*}\right) \in \operatorname{dom} f$. By 
Proposition 2[(iv)], $\left(2 x^{*}, 2 y^{*}\right) \in \operatorname{dom} \phi^{*} \subset X \times C$ and hence $2 y^{*} \in C$. Using Proposition 2[(v)] we deduce that

$$
\begin{aligned}
0 & =4\left\langle x^{*} \mid y^{*}\right\rangle+\left\|y^{*}-x^{*}\right\|^{2}-\left\|x^{*}+y^{*}\right\|^{2} \\
& =p\left(2 x^{*}, 2 y^{*}\right)+\left\|y^{*}-x^{*}\right\|^{2}-\left\|\left(y^{*}-x^{*}\right)-2 y^{*}\right\|^{2} \\
& \leq \phi^{*}\left(2 x^{*}, 2 y^{*}\right)+\left\|y^{*}-x^{*}\right\|^{2}-d_{C}^{2}\left(y^{*}-x^{*}\right) \\
& =2\left(f\left(x^{*}, y^{*}\right)+g\left(y^{*}-x^{*}\right)\right) \\
& =2\left(f\left(x^{*}, y^{*}\right)+g\left(L\left(x^{*}, y^{*}\right)\right)\right) .
\end{aligned}
$$

Hence $\inf (f+g L)(X \times X) \geq 0$ and, since $\operatorname{dom} g=X$, Fact 3 now implies that

$$
\min _{x \in X} f^{*}\left(-L^{*} x\right)+g^{*}(x) \leq 0 .
$$

Since $f^{*}=\frac{1}{2} \phi, g^{*}=j+\iota_{C}$, and $L^{*}: X \rightarrow X \times X: x \mapsto(-x, x)$, we see that (13) clearly yields (7).

Now assume that $y \neq 0$. Let $E=D-y$ and define $U: E \rightarrow X: z \mapsto T(z+y)$. Then $U$ is firmly nonexpansive and $U(E)=T(D)$. By what we just proved, there exists an extension $\widetilde{U}: E \cup\{0\} \rightarrow \overline{\operatorname{conv}} U(E)=\overline{\operatorname{conv}} T(D)$. Therefore, $\widetilde{T}: D \cup\{y\} \rightarrow$ $\overline{\operatorname{conv}} T(D): z \mapsto \widetilde{U}(z-y)$ is as required.

Corollary 5. Let $D$ be a nonempty subset of $X$ and let $T: D \rightarrow X$ be firmly nonexpansive. Then $T$ has a firmly nonexpansive extension $\widetilde{T}: X \rightarrow \overline{\operatorname{conv}} T(D)$.

Proof. Let $\mathcal{M}$ be the set of all pairs $(U, E)$, where $D \subset E \subset X$ and $U: E \rightarrow$ $\overline{\text { conv }} T(D)$ is a firmly nonexpansive extension of $T$. Partially order $\mathcal{M}$ via $\left(U_{1}, E_{1}\right) \preceq$ $\left(U_{2}, E_{2}\right)$ if $E_{1} \subset E_{2}$ and $U_{2}$ extends $U_{1}$. Zorn's lemma guarantees the existence of a maximal element $(\widetilde{T}, \widetilde{D})$. Now Theorem 4 shows that $\widetilde{D}=X$.

Remark 6 (range localization is optimal). The conclusion that the range of the extension $\widetilde{T}$ lies in the closed convex hull of $T(D)$ cannot be improved upon in general. Indeed, let $D$ be a nonempty subset of $X$, let $T$ be Id $\left.\right|_{D}$, and let $\widehat{T}: X \rightarrow X$ be any firmly nonexpansive extension of $T$. Then $D=\operatorname{Fix} T \subset \operatorname{Fix} \widehat{T}$, and the last set is closed and convex [13, 14. Hence $C=\overline{\operatorname{conv}} T(D)=\overline{\operatorname{conv}} D \subset \operatorname{Fix} \widehat{T} \subset \widehat{T}(X)$. In particular, let $\widetilde{T}: X \rightarrow C$ be any firmly nonexpansive extension of $T$ as in Corollary [5. Then $\widetilde{T}(X)=C$ and $\widetilde{T}|C=\operatorname{Id}|_{C}$; therefore, $\widetilde{T}$ is the projector onto $C$.

Corollary 7 (Kirszbraun-Valentine). Let $D$ be a nonempty subset of $X$ and let $N: D \rightarrow X$ be nonexpansive. Then $N$ has a nonexpansive extension $\tilde{N}: X \rightarrow$ $\overline{\mathrm{conv}} N(D)$.

Proof. (See also [13, 16, 20, 26, for different proofs and related results.) Let $T=\left.\frac{1}{2} \operatorname{Id}\right|_{D}+\frac{1}{2} N$, which is firmly nonexpansive. Corollary 5 guarantees a firmly nonexpansive extension $\widetilde{T}: X \rightarrow \overline{\operatorname{conv}} T(D)$. Let $P$ be the (firmly) nonexpansive projector onto $\overline{\operatorname{conv}} N(D)$. Then $\widetilde{N}=P \circ(2 \widetilde{T}-\mathrm{Id})$ is as required.

Remark 8. We do not know whether it is possible to deduce Corollary 5 from Corollary 7 . The following technique of going back and forth between firmly nonexpansive and nonexpansive mappings, utilized in the proof of Corollary 7, does not work in reverse. Let $D$ be a nonempty subset of $X$ and $T: D \rightarrow X$ be firmly nonexpansive. Then $N=2 T-\left.\operatorname{Id}\right|_{D}: D \rightarrow X$ is nonexpansive, and hence (by 
Corollary 17) it has an extension $\widetilde{N}: X \rightarrow \overline{\operatorname{conv}} N(D)$. It is tempting to conjecture that $\widetilde{T}=\frac{1}{2} \mathrm{Id}+\frac{1}{2} \widetilde{N}$ would be an extension of $T$ as in Corollary 5 . However, let us concretely consider $D=\{0\} \subset X$ and $T: D \rightarrow X: 0 \mapsto 0$. Then $N=2 T-\operatorname{Id}_{D}=T$ and so $\widetilde{N} \equiv 0$. Hence $\widetilde{T}=\frac{1}{2}$ Id, which does not satisfy $\widetilde{T}(X) \subset \overline{\operatorname{conv}} T(D)=\{0\}$.

Remark 9. The correspondence revealed by Minty [18 between (maximal) monotone operators and firmly nonexpansive mappings (with full domain) provides a reformulation of Theorem 4 in terms of monotone operators (see, e.g., 6, Theorem 2.1]), which in turn relates to the work of Debrunner and Flor 11. The new proof presented here provides a convex-analytical handle on these results (see also [4]). Furthermore, in the present Hilbert space setting, Reich (21, Lemma 2.1]) showed that Corollary 5 is equivalent to the following result. Let $A$ be a monotone operator on $X$ with a nonempty graph. Then $A$ has a maximal monotone extension $\widetilde{A}$ such that $\overline{\operatorname{conv}} \operatorname{dom} A=\overline{\operatorname{conv}} \operatorname{dom} \widetilde{A}$. (In fact, his result is about accretive operators in general Banach spaces.) Using [21, Proposition 2.2], it follows that Corollary 5 actually characterizes Hilbert spaces among all Banach spaces of dimension not less than three.

\section{ACKNOWLEDGMENT}

The author wishes to thank the referee for his insightful comments. H. H. Bauschke's work was partially supported by the Natural Sciences and Engineering Research Council of Canada.

\section{REFERENCES}

[1] H. H. Bauschke and J. M. Borwein, "On projection algorithms for solving convex feasibility problems," SIAM Review, vol. 38, pp. 367-426, 1996. MR1409591 (98f:90045)

[2] H. H. Bauschke and P. L. Combettes, "A weak-to-strong convergence principle for Fejérmonotone methods in Hilbert spaces," Mathematics of Operations Research, vol. 26, pp. 248264, 2001. MR.1895827(2003f:65101)

[3] H. H. Bauschke, P. L. Combettes, and S. Reich, "The asymptotic behavior of the composition of two resolvents," Nonlinear Analysis: Theory, Methods, and Applications, vol. 56, pp. 283301, 2005. MR2101879 (2006d:47088)

[4] J. M. Borwein, "Maximal monotonicity via convex analysis," to appear in Journal of Convex Analysis.

[5] J. M. Borwein and Q. J. Zhu, Techniques of Variational Analysis, Springer-Verlag, 2005. MR.2144010

[6] H. Brézis, Opérateurs maximaux monotones et semi-groupes de contractions dans les espaces de Hilbert, North-Holland, 1973. MR0348562 (50:1060)

[7] R. E. Bruck and S. Reich, "Nonexpansive projections and resolvents of accretive operators in Banach spaces," Houston Journal of Mathematics, vol. 3, pp. 459-470, 1977. MR0470761 $(57: 10507)$

[8] Y. Censor and S. Reich, "Iterations of paracontractions and firmly nonexpansive operators with applications to feasibility and optimization," Optimization, vol. 37, pp. 323-339, 1996. MR.1402641 (98j:47161)

[9] P. L. Combettes, "Construction d'un point fixe commun à une famille de contractions fermes," Comptes Rendus des Séances de l'Académie des Sciences, Série I, Mathématique, vol. 320, pp. 1385-1390, 1995. MR1338291 (96c:47087)

[10] P. L. Combettes, "Solving monotone inclusions via compositions of nonexpansive averaged operators," Optimization, vol. 53, pp. 475-504, 2004. MR2115266 (2005i:47088)

[11] H. Debrunner and P. Flor, "Ein Erweiterungssatz für monotone Mengen," Archiv der Mathematik, vol. 15, pp. 445-447, 1964. MR0170189(30:428) 
[12] S. Fitzpatrick, "Representing monotone operators by convex functions," Workshop/Miniconference on Functional Analysis and Optimization (Canberra 1988), Proceedings of the Centre for Mathematical Analysis, Australian National University vol. 20, Canberra, Australia, pp. 59-65, 1988. MR.1009594 (90i:47054)

[13] K. Goebel and W. A. Kirk, Topics in Metric Fixed Point Theory, Cambridge University Press, 1990. MR.1074005 (92c:47070)

[14] K. Goebel and S. Reich, Uniform Convexity, Hyperbolic Geometry, and Nonexpansive Mappings, Marcel Dekker, 1984. MR0744194 (86d:58012)

[15] W. Kaczor, "Fixed points of asymptotically regular nonexpansive mappings on nonconvex sets," Abstract and Applied Analysis, vol. 2003, pp. 83-91, 2003. MR1960139 (2004a:47066)

[16] M. D. Kirszbraun, "Über die zusammenziehende und Lipschitzsche Transformationen," Fundamenta Mathematicae, vol. 22, pp. 77-108, 1934.

[17] K. C. Kiwiel and B. Łopuch, "Surrogate projection methods for finding fixed points of firmly nonexpansive mappings," SIAM Journal on Optimization, vol. 7, pp. 1084-1102, 1997. MR.1479616 (98j:90049)

[18] G. J. Minty, 'Monotone (nonlinear) operators in Hilbert space," Duke Mathematical Journal, vol. 29, pp. 341-346, 1962. MR0169064 (29:6319)

[19] J.-J. Moreau, "Proximité et dualité dans un espace hilbertien," Bulletin de la Société Mathématique de France, vol. 93, pp. 273-299, 1965. MR0201952 (34:1829)

[20] S. Park, "Generalized Kirszbraun-Minty type inequalities," in Fixed point theory and applications, pp. 197-203, Nova Science Publishers, 2002. MR2083504 (2005d:49017)

[21] S. Reich, "Extension problems for accretive sets in Banach spaces," Journal of Functional Analysis, vol. 26, pp. 378-395, 1977. MR0477893 (57:17393)

[22] S. Reich and I. Shafrir, "The asymptotic behavior of firmly nonexpansive mappings," Proceedings of the American Mathematical Society, vol. 101, pp. 245-250, 1987. MR0902536 (88i:47030)

[23] S. Reich and S. Simons, "Fenchel duality, Fitzpatrick functions and the Kirszbraun-Valentine extension theorem," Proceedings of the American Mathematical Society, vol. 133, pp. 26572660, 2005. MR2146211 (2006d:46025)

[24] S. Simons and C. Zălinescu, "Fenchel duality, Fitzpatrick functions and maximal monotonicity," Journal of Nonlinear and Convex Analysis, vol. 6, pp. 1-22, 2005. MR2138099 (2005k:49102)

[25] P. Tseng, "On the convergence of the products of firmly nonexpansive mappings," SIAM Journal on Optimization, vol. 2, pp. 425-434, 1992. MR1172499 (93f:90161)

[26] F. A. Valentine, "On the extension of a vector function so as to preserve a Lipschitz condition," Bulletin of the American Mathematical Society, vol. 49, pp. 100-108, 1943. MR0008251 $(4: 269 \mathrm{~d})$

[27] C. Zălinescu, Convex Analysis in General Vector Spaces, World Scientific Publishing, 2002. MR1921556 (2003k:49003)

Department of Mathematics, Irving K. Barber School, University of British Columbia Okanagan, Kelowna, British Columbia, Canada V1V 1V7

E-mail address: heinz.bauschke@ubc.ca 